\title{
Constitutional Perspectives on Machine Learning
}

\author{
Harsh Gupta \\ Indian Insitute of Technology, Kharagpur
}

\begin{abstract}
This article discusses the notions of individual fairness and group fairness discussed in the fairness, accountability and transparency in machine learning (FATML) literature, in the light of equality and anti-discrimination provisions in The Constitution of India.
\end{abstract}

Recent year have seen an exponential rise in the use of machine learning and algorithmic systems in almost all domains, including very "human" aspects of human society. Now algorithmic systems suggests us which music should we listen to, which movie to watch, which news article is worth reading, which resumes to forward to the hiring manager, who should be given admission and even who should be given a larger sentence for a crime. Historically some of these areas, like employment, education and crime have seen a lot of contention regarding bias and discrimination.

Often algorithmic systems are projected as something which is neutral and has the ability to fix human bias. But recent experiences have been shown that they can not only reflect human biases but also exaggeration it. Large amount of literature is emerging which brings out more of these biases, finds out why these biases occur and how to tackle them. These algorithms takes decisions in real world, affecting people real people in real ways, therefore I believe their behavior needs to regulated to prevent harm.

\section{Motivating Examples}

\section{Racial in Crime Risk Assessment}

Some jurisdictions in the United States use a software system to assess the risk of a re-offending by an offender. A study by ProPublica found that though the accuracy of the system for whites and blacks was similar, the type of error they make for different races are very different. Blacks are almost twice as likely as whites to be labeled a higher risks but not actually re-offend. The system made the opposite mistake for whites. [1] 


\begin{tabular}{lcc}
\hline Error type & White & Black \\
\hline Labeled Higher Risk, But Didn't Re-Offend & $23.5 \%$ & $44.9 \%$ \\
Labeled Lower Risk, Yet Did Re-Offend & $47.7 \%$ & $28.0 \%$ \\
\hline
\end{tabular}

This particular reported has generated wide response, including rebuttal from Northpoint, the company which makes the risk assessment software and rebuttal of rebuttal from ProPublica. The main argument from Northpoint's side is that rate of violent crimes is higher among blacks directly leads to asymmetry in the two error rates and correcting for the disparity in error rates will lead to fair treatment to white inmates [2]. Recent literature, has suggested that it might be impossible to achieve certain notions of fairness without compromising on others. [3]

\section{Blacks being tagged as Gorillas}

Users found that in a Machine Learning based image tagging system in Google Photos produced offensive labels, tagging black people in photos as "gorillas". [4] In a similar example, flickr was found to tag a black person as 'Ape' [5] and in 2009 it was seen that when HP's web cam wasn't able to recognize black faces when it was easily able to track white ones [6] Though there are technical explanation for these behaviors of the algorithms, Face recognition techniques are used widely to identify offenders and error or bias in these systems can lead to loss of reputation and livelihood of innocent people. [7]

\section{Occupational Gender stereotypes in Google Images}

The current society has large amount of gender bias with regards to occupations. It was found that Google Image search results exaggerate the existing gender bias in occupation. This is important because representation of gender in image search results shifts people's perception about real world distribution. [8]

\section{Staples Dynamic Pricing}

An online shopping store in US, Staples dynamically price items for customers on the basis of their browsing history, location from the nearest competitor store and other parameters. A study by The Wall Street Journal found that the pricing scheme worked negatively for low-income households as they are more likely to live outside the core of the city. This considered as an unintended side effect of the pricing scheme. [9] 


\section{Job Advertisements}

A study on effects of web tracking on advertisements by Google found that setting the gender to female resulted in fewer instances of advertisements related to high paying jobs. [10]

\section{Overview of reasons of Biases}

\section{Poorly Collected or Poorly Selected Data}

Good algorithms require good data to work on it. The source of data can have inherent biases, for example the typical editor of Wikipedia is someone who is White, college educated, western and male [11] [12]. The content of wikipedia also under represents non western population of the world. [13]

The data get corrupted while handing and transformation, or though bugs in software [14].

The process of collecting data can also introduce certain bias in the data, for example if certain data is collected through online form, people with low access to the internet are likely to missing from the data, and these people are also more likely to be low income, less educated, non English speaking and from depressed race and caste. The errors introduced through selection process can be very subtle, such disparities which are often under appreciated.

\section{Poor Algorithm}

Even if the underlying data is of good quality and represents the population well, the design of the algorithm might introduce certain biases in the result. One example such effects is called uncertainty bias.

The uncertainty associated with a subpopulation in data has inverse relation with the sample. In cases the algorithmic system takes decisions conservatively, uncertainty bias can lead to inferior result for population with lower sample, even if everything else remains same.

To illustrate this consider you have a sample 1000 people, 900 white and 100 black and all of them have same likelihood of defaulting on a loan $5 \%$, and you give loan to any with a default rate of less $10 \%$. Now if you give loans conservatively (if you think chances of someone defaulting is between 3 to $8 \%$ then you'll consider defaulting rate to be $8 \%$ ) you won't give loans to any of the black folks and to every white folk and that because their sample size is smaller the uncertainty associated with black sample is higher than the white sample. $[15]$ 


\section{FATML: Fairness, Accountability and Trans- parency in Machine Learning}

There is a growing area of research often known as "Fairness, Accountability and Transparency in Machine Learning (FATML)" looks at some of issues described above through a computational perspective.

Two important notions of fairness which are used in the FATML literature are that of "group fairness" and "individual fairness".

A system is group fair to certain group attributes, say caste, gender or race, if the overall output of the system doesn't differ more than a certain threshold for the groups defined through the sensetive attribute.

A system is defined to be individual fair, if and only if the output for two individuals who are similar in the relevant characteristics are similar.

The literature gives several methods to maintain the property of group and individual fairness in machine learning systems. Recent studies also found out unless certain special conditions are met, there are fundamental tradeoffs between maintaining group and individual fairness and in general is possible to maintain both.

\section{Fairness and anti-discrimination in The Consti- tution of India}

Part of III of The Constitution of India specifies the Fundamental Rights given to every citizen of India. Of then Article 14 describes right to equality, Article 15 formulates the anti-discrimination provisions and Article 16 talks about antidiscrimination in terms of public employment by the government. Though these provisions apply mostly to actions by State, these cover a large portion real world issues which can occur though machine discrimination.

Corporations largely owned and funded by the Government are also covered under these provisions.

\section{Article 14}

Article 14 of The Constitution of India gives every citizen the right to equality before law. It says:

The State shall not deny to any person equality before law or equal protection of the laws withing the territory of India. 
Equality here doesn't mean that every citizen should be treated in the exact same matter but is understood as similarly situated persons should be treated similarly. To determine when State can make different treatment, the Courts have formulated what is called the test of reasonable classification. The State can treat two persons differently only if:

1. There is an intelligible differential between the two persons

2. There is an objective for the different treatment and the different treatment satisfies the objective.

Another way test of resonable classification is stated is "similarly situtated people should be treated similarly" which is similar to the individual fairness definition used in FATML literature.

This is not to say only the test of reasonable classification determines equality in context of Article 14. Court have used various legal and philosophical principles to determine when a particular action of State is to considered equal. But test of reasonable classification is to understood as a minimum criterion for equality. Because of this Article 14 can be generally to used to seek remedy for discriminated against by algorithmic system used by State.

Courts have also said that the reason for discrimination in a state action must be disclosed, applied to algorithmic systems, this is can lead to an effect similar to "Right to explanation" provision under European Union's General Data Protection Regulation (GDPR)[15]. GDPR, after coming into force in 2018, will allow citizens of the member countries to seek explanation in cases where an algorithmic systems make a siginificant decision about them.

\section{Article 15(1)}

Article 15(1) says:

The State shall not discriminate against any citizen only on the grounds of religion, race, caste, sex, place of birth or any of them.

The word "only" is important here, Article 15(1) doesn't guarantee equality of outcome of these different groups but says that while considering whether two people are similarly or situated or not sex, religion, race, caste or place of birth.

As it has been widely studied in the context of racial bias in United States, ignoring the race doesn't eliminate racial discrimination as wide array of useful information, from someone's pin code to the name is correlated with race. What is not clear is that to what extent Article 15(1) forces one to normalize these parameters in terms of race. 
Both Article 14 and Article 15(1) works towards ensuring individual fairness but as we have discussed in the section above doing so often disallows us to work towards group level fairness. Interestingly, provisions to Article 15(1), that is $15(3), 15(4)$ and $15(5)$ allows state to override individual fairness mechanism to ensure group level fairness. They read as follows:

(3) Nothing in this article shall prevent the State from making any special provision for women and children.

(4) Nothing in this article or in clause (2) of article 29 shall prevent the State from making any special provision for the advancement of any socially or educationally backward classes of citizens or for the Scheduled Castes and the Scheduled Tribes.

(5) Nothing in this article or in sub-clause (g) of clause (1) of article 19 shall prevent the State from making any special provision, by law, for the advancement of any socially and educationally backward classes of citizens or for the Scheduled Castes or the Scheduled Tribes in so far as such provisions relate to their admission to educational institutions including private educational institutions, whether aided or unaided by the State, other than the minority educational institutions referred to in clause (1) of article 30.

\section{References}

[1] J. Angwin, J. Larson, S. Mattu, and K. Kirchner, "Machine Bias: There's Software Used Across the Country to Predict Future Criminals. And it's Biased Against Blacks." ProPublica. May-2016 [Online]. Available: https://www. propublica.org/article/machine-bias-risk-assessments-in-criminal-sentencing. [Accessed: 11-Nov-2016]

[2] "A computer program used for bail and sentencing decisions was labeled biased against blacks. It's actually not that clear." Washington Post. Oct-2016 [Online]. Available: https://www.washingtonpost.com/news/monkey-cage/wp/ 2016/10/17/can-an-algorithm-be-racist-our-analysis-is-more-cautious-than-propublicas/. [Accessed: 12-Nov-2016]

[3] J. Kleinberg, S. Mullainathan, and M. Raghavan, "Inherent Trade-Offs in the Fair Determination of Risk Scores," arXiv:1609.05807 [cs, stat], Sep. 2016 [Online]. Available: http://arxiv.org/abs/1609.05807. [Accessed: 14-Nov-2016]

[4] "Google Photos labeled black people 'gorillas'," USA TODAY. 2015 [Online]. Available: http://www.usatoday.com/story/tech/2015/07/01/ 
google-apologizes-after-photos-identify-black-people-as-gorillas /29567465/.

[Accessed: 11-Nov-2016]

[5] "Flickr Fixing 'Racist' Auto-Tagging Feature After Black Man Mislabeled 'Ape'," PetaPixel. May-2015 [Online]. Available: http://petapixel.com/2015/ 05/20/flickr-fixing-racist-auto-tagging-feature-after-black-man-mislabeled-ape/. [Accessed: 12-Nov-2016]

[6] A. Frucci, "HP Face-Tracking Webcams Don't Recognize Black People," Gizmodo. 2009 [Online]. Available: http://gizmodo.com/5431190/ hp-face-tracking-webcams-dont-recognize-black-people. [Accessed: 12-Nov2016]

[7] A. Kofman, "How a Facial Recognition Mismatch Can Ruin Your Life," The Intercept. 2016 [Online]. Available: https://theintercept.com/2016/10/ 13/how-a-facial-recognition-mismatch-can-ruin-your-life/. [Accessed: 09-Nov2016]

[8] M. Kay, C. Matuszek, and S. A. Munson, "Unequal Representation and Gender Stereotypes in Image Search Results for Occupations," in Proceedings of the 33rd Annual ACM Conference on Human Factors in Computing Systems, 2015, pp. 3819-3828 [Online]. Available: http://doi.acm.org/10.1145/2702123. 2702520. [Accessed: 09-Nov-2016]

[9] J. Valentino-DeVries, J. Singer-Vine, and A. Soltani, "Websites Vary Prices, Deals Based on Users' Information," Wall Street Journal, Dec. 2012 [Online]. Available: http://www.wsj.com/articles/SB10001424127887323777204578189391813881534. [Accessed: 11-Nov-2016]

[10] A. Datta, M. C. Tschantz, and A. Datta, "Automated Experiments on Ad Privacy Settings," Proceedings on Privacy Enhancing Technologies, vol. 2015, no. 1, pp. 92-112, 2015 [Online]. Available: https://www.degruyter.com/view/j/popets.2015.1.issue-1/popets-2015-0007/ popets-2015-0007.xml?ncid=txtlnkusaolp00000618. [Accessed: 11-Nov-2016]

[11] "Wikipedia Survey - Overview of Results." Mar-2010 [Online]. Available: https://web.archive.org/web/20110728182835/http://www.wikipediastudy. org/docs/Wikipedia_Overview_15March2010-FINAL.pdf. [Accessed: 14-Nov2016]

[12] S. T. K. Lam, A. Uduwage, Z. Dong, S. Sen, D. R. Musicant, L. Terveen, and J. Riedl, "WP: Clubhouse?: An exploration of Wikipedia's gender imbalance," in Proceedings of the 7th international symposium on Wikis and open collaboration, 2011, pp. 1-10 [Online]. Available: http://dl.acm.org/citation. cfm?id=2038560. [Accessed: 14-Nov-2016]

[13] M. Graham, R. K. Straumann, and B. Hogan, "Digital Divisions of Labor and Informational Magnetism: Mapping Participation in Wikipedia," $A n$ nals of the Association of American Geographers, vol. 105, no. 6, pp. 11581178, Nov. 2015 [Online]. Available: http://dx.doi.org/10.1080/00045608.2015. 1072791. [Accessed: 14-Nov-2016] 
[14] J. Hrala, "Excel Is to Blame for Major Typos in 20\% of Scientific Papers on Genes," ScienceAlert. Aug-2016 [Online]. Available: http://www.sciencealert. com/excel-is-responsible-for-20-percent-of-errors-in-genetic-scientific-papers.

[Accessed: 14-Nov-2016]

[15] B. Goodman and S. Flaxman, "European Union regulations on algorithmic decision-making and a' right to explanation'," arXiv preprint arXiv:1606.08813, 2016 [Online]. Available: https://arxiv.org/abs/1606.08813. [Accessed: 09-Nov2016] 